\title{
Interfacial operator approach to computing modes of surface plasmon polaritons for periodic structures
}

\author{
Chien C. Chang, ${ }^{1,2, *}$ R. L. Chern, ${ }^{2}$ C. Chung Chang, ${ }^{1}$ and R. R. Hwang ${ }^{3}$ \\ ${ }^{1}$ Division of Mechanics, Research Center for Applied Sciences, Academia Sinica, Taipei 115, Taiwan, Republic of China \\ ${ }^{2}$ Institute of Applied Mechanics, National Taiwan University, Taipei 106, Taiwan, Republic of China \\ ${ }^{3}$ Department of System Engineering and Naval Architecture, National Taiwan Ocean University, \\ Keelung 202, Taiwan, Republic of China
}

(Received 3 August 2005; published 9 November 2005)

\begin{abstract}
It has been difficult to compute the band structures for photonic crystals with metallic components included in the periodic units. The existence of modes of surface plasmon polariton presents the major difficulty not only because of the localized nature of the modes but also of the apparent necessity of handling a nonlinear eigenvalue problem. Here we show that by introducing an interfacial operator within the finite-difference framework, we are able to formulate the problem for computing modes of surface plasmon polariton in the format of standard eigenvalue problems. Results are uncovered by applying the method to periodic structures with corrugated interfaces between metals and dielectric materials, as well as other classes of interfaces.
\end{abstract}

DOI: 10.1103/PhysRevB.72.205112

PACS number(s): 42.70.Qs, 02.70.Bf, 78.20.Bh

\section{INTRODUCTION}

The optical properties of a photonic crystal are dependent upon the comprising materials. Most previous studies in this area of research are concerned with the optical properties of a periodic structure comprising two or more dielectric materials. Recently, we have seen a steadily growing interest in considering the effects of including metallic components in photonic crystals. In particular, the interaction of light with metals in a periodic structure excites the modes of surface plasmon polariton ${ }^{1,2}$ and causes possible band gaps in the dispersion relations. ${ }^{3,4}$ This enables us to explore plasmonic crystals or plasmonic band gap materials made of metallic materials in a similar way to photonic crystals or photonic band gap materials made of dielectric materials.

In considering the real metal effects for a photonic crystal, such as the free-electron model $\varepsilon_{m}(\omega)=1-\omega_{p}^{2} / \omega^{2}$, where $\omega_{p}$ denotes the bulk plasma frequency, the dielectric function is frequency dependent, and therefore for a given wave vector the solution of the dispersion relation or band structure apparently poses a nonlinear eigenvalue problem as the eigenfrequency itself appears in the solution operator. ${ }^{5}$ It is known that surface plasmon polaritons are evanescent TE modes, living in the neighborhood of the interface of the metallic inclusion and the surrounding dielectric material. This is only possible if $\omega<\omega_{p}$, that is, when the dielectric function of the metallic components is in the opposite sign to that of the surrounding dielectric material. The latter means that in mathematical terms, the equation would change type as one crosses the interface from the dielectric to the metal, and vice versa. The localized nature of surface plasmon polaritons requires fine resolution near the interface, and the change of type of the equation makes the problem nondefinite that causes numerical difficulties for many solvers such as the method of conjugate gradient. ${ }^{6}$ Nevertheless, a few methods have been applied for this frequency-dependent nonlinear eigenvalue problem. The method of multiple multipole expansion $^{7}$ is another choice in which a careful definition of cost function is required for detecting resonant frequencies. The method of solution for eigenfrequencies by detecting resonance peaks is also applicable to the finite-difference time-domain method. ${ }^{8} \mathrm{~A}$ theory of complex resonances was introduced for the general absorptive periodic dielectric structures; the resonances define the eigenfrequencies. ${ }^{9-11}$ Along this line, a specific perturbation theory was also developed with mathematical rigor to study the spectral properties of absorptive photonic crystals. ${ }^{12}$ Also, we have seen the method of vectorial eigenmode expansion applied to study phonon-polariton excitation in photonic crystals. ${ }^{13}$

In this study, we present a direct method for obtaining the modes of surface plasmon polariton as well as other branches of modes. This is achieved by considering Maxwell's equations for the metallic and dielectric regions separately and treating the interface by an interfacial operator that passes information between both sides of the interface. In particular, within the finite-difference framework, we are able to formulate the problem for computing modes of surface plasmon polaritons in the format of standard eigenvalue problems. The method will first be illustrated for a onedimensional periodic array of alternating layers of metals and dielectrics, as shown in Fig. 1, and then we show how the method can be extended to problems in two space dimensions. Perturbation analysis is also included to consider the effect of Drude damping.

\section{INTERFACIAL OPERATOR}

For linear isotropic and nonmagnetic dielectric materials, the equation for time-harmonic TM modes in two dimensions can be written as

$$
-\frac{1}{\varepsilon} \nabla^{2} E=\left(\frac{\omega}{c}\right)^{2} E
$$

where $\varepsilon=\varepsilon(\mathbf{r})$ is the dielectric function. Let $\varepsilon=\varepsilon_{d}$ in the dielectric, and $\varepsilon=\varepsilon_{m}$ in the metal. Consider the free-electron 


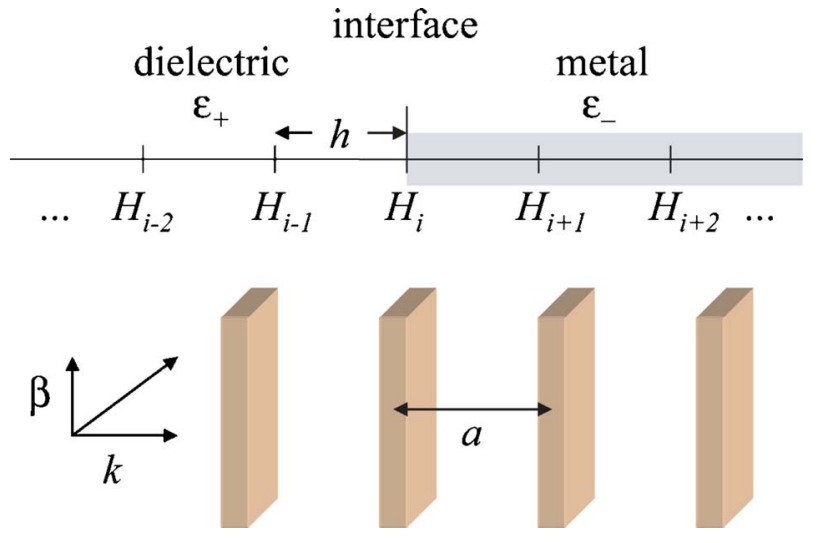

FIG. 1. (Color online) Discretization points of one unit cell for a one-dimensional periodic array of alternating layers of metals and dielectrics, where $\beta$ is the off-line wave number.

model $\varepsilon_{m}=1-\omega_{p}^{2} / \omega^{2}$. In the metal, Eq. (1) becomes

$$
-\nabla^{2} E=\left[\left(\frac{\omega}{c}\right)^{2}-\left(\frac{\omega_{p}}{c}\right)^{2}\right] E
$$

or, equivalently,

$$
\left[-\nabla^{2}+\left(\frac{\omega_{p}}{c}\right)^{2}\right] E=\left(\frac{\omega}{c}\right)^{2} E
$$

The present authors developed a fast inverse method with multigrid acceleration ${ }^{14}$ to solve Eq. (3). However, this cannot be done for TE modes

$$
-\nabla \cdot\left(\frac{1}{\varepsilon} \nabla H\right)=\left(\frac{\omega}{c}\right)^{2} H
$$

But in the strict insides of the dielectric and the metal, we can move $1 / \varepsilon$ outside the operator $-\nabla$, and still obtain

$$
-\frac{1}{\varepsilon_{d}} \nabla^{2} H=\left(\frac{\omega}{c}\right)^{2} H
$$

and

$$
\left[-\nabla^{2}+\left(\frac{\omega_{p}}{c}\right)^{2}\right] H=\left(\frac{\omega}{c}\right)^{2} H .
$$

First of all, let us discretize Eqs. (5) and (6) in the strict insides of the dielectrics and metal by a central finite difference with the interface point $H_{i}$ as the boundary value. The lattice with lattice constant $a$ is partitioned into $n$ equal segments with $n$ independent variables, as shown in Fig. 1, because the $(n+1)$ th variable is related to the first variable by Bloch's condition

$$
H_{n+1}=e^{i k a} H_{1} \text {. }
$$

We obtain a system of linear equations with $\Lambda=(\omega / c)^{2}$,

$$
\widetilde{\mathbf{A}}_{n-1 \times n} \widetilde{\mathbf{H}}_{n}=\Lambda \widetilde{\mathbf{H}}_{n-1},
$$

where $\widetilde{\mathbf{H}}_{n}=\left[\begin{array}{lllllll}H_{1} & \cdots & H_{i-1} & H_{i} & H_{i+1} & \cdots & H_{n}\end{array}\right]^{T}$, and $\widetilde{\mathbf{H}}_{n-1}$ is obtained by deleting $H_{i}$ from $\widetilde{\mathbf{H}}_{n}$. This does not constitute an eigensystem because $\widetilde{\mathbf{A}}$ is not a square matrix and one missing equation is needed. This is provided by applying Eq. (4) at the interface to relate the normal derivatives on both sides as

$$
\left.\frac{1}{\varepsilon_{+}} \frac{\partial H}{\partial n}\right|_{+}=\left.\frac{1}{\varepsilon_{-}} \frac{\partial H}{\partial n}\right|_{-}
$$

Then, we have, with $\varepsilon_{+}=\varepsilon_{d}$ and $\varepsilon_{-}=1-\omega_{p}^{2} / \omega^{2}$

$$
\left.\frac{\partial H}{\partial n}\right|_{+}=\frac{\omega^{2}}{\omega_{p}^{2}}\left(\left.\frac{\partial H}{\partial n}\right|_{+}-\left.\varepsilon_{d} \frac{\partial H}{\partial n}\right|_{-}\right) \text {. }
$$

Nevertheless, Eqs. (6) and (10) do not constitute an easy eigenvalue problem because the variable in Eq. (6) is $H$, while the one in Eq. (10) would be $\partial H /\left.\partial n\right|_{+}-\varepsilon_{d} \partial H /\left.\partial n\right|_{-}$.

The crucial step taken here is to introduce an interfacial variable into the discretized form of Eq. (10), and reformulate the eigensystem in terms of the $H$ field in the strict insides along with this new variable. Applying one-sided finite difference to Eq. (10) at the interface point $i$, we have, with $\Lambda_{p}=\left(\omega_{p} / c\right)^{2}$

$$
\Lambda_{p}\left(\frac{H_{i}-H_{i-1}}{h}\right)=\Lambda\left[\frac{-H_{i-1}+\left(1+\varepsilon_{d}\right) H_{i}-\varepsilon_{d} H_{i+1}}{h}\right] .
$$

This suggests that we introduce an interfacial variable

$$
R_{i} \equiv-H_{i-1}+\left(1+\varepsilon_{d}\right) H_{i}-\varepsilon_{d} H_{i+1}
$$

or, reversely,

$$
H_{i}=\frac{-H_{i-1}+R_{i}-H_{i+1}}{1+\varepsilon_{d}}
$$

so that Eq. (11) can be recast into the form

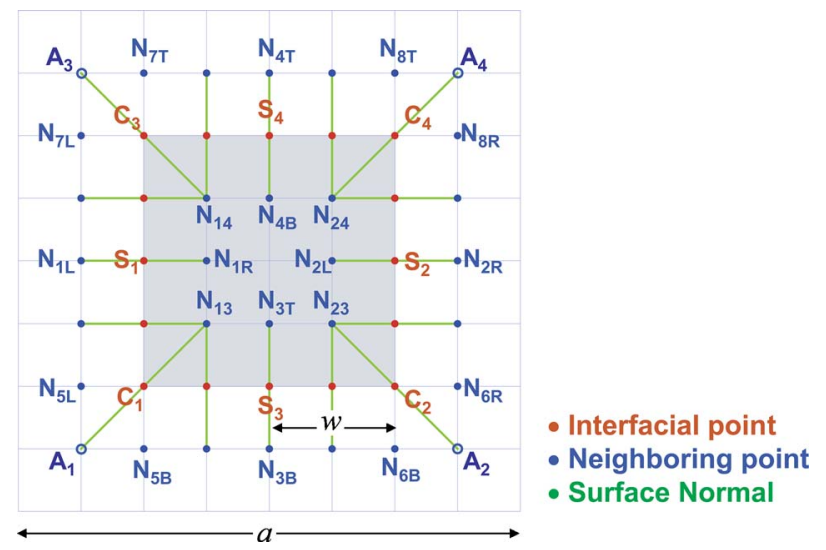

FIG. 2. (Color online) Interfacial and neighboring points for the square lattice of square metal cylinders with lattice constant $a$ and half width $w / a=0.25$. 


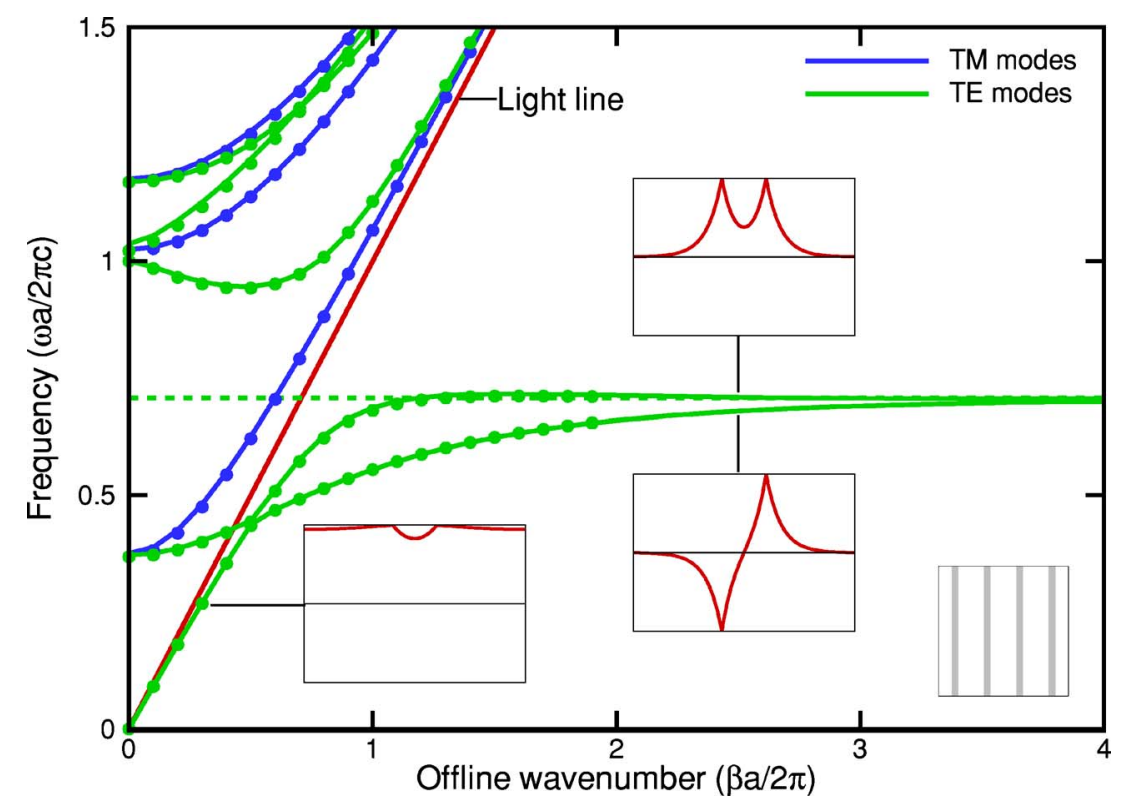

FIG. 3. (Color online) Dispersion relations at $k=0$ for the onedimensional photonic crystal with $d_{2} / a=0.2$. Solid lines denote computed results by the interfacial operator approach and dots denote analytical solutions of Eq. (35) by Newton-Ralphson's method.

$$
\frac{\Lambda_{p}}{1+\varepsilon_{d}}\left(-\varepsilon_{d} H_{i-1}+R_{i}+\varepsilon_{d} H_{i+1}\right)=\Lambda R_{i} .
$$

Meanwhile, we rewrite the matrix Eq. (8) by using Eq. (13) to replace each $H_{i}$ at the interface. The resultant matrix system supplemented by Eq. (14) constitutes an eigensystem of standard format

$$
\mathbf{A}_{n \times n} \mathbf{H}_{n}=\Lambda \mathbf{H}_{n},
$$

where $\mathbf{H}_{n}=\left[H_{1} \cdots H_{i-1} R_{i} H_{i+1} \cdots H_{n}\right]^{T}$. Then, more explicitly, we have the entries of the rows corresponding to $H_{i-1}, R_{i}$, and $H_{i+1}$

$$
\mathbf{A}=\frac{1}{h^{2}}\left[\begin{array}{ccccc}
\cdots & & & & \cdots \\
-1 & \frac{1+2 \varepsilon_{d}}{1+\varepsilon_{d}} & -\frac{1}{1+\varepsilon_{d}} & -\frac{\varepsilon_{d}}{1+\varepsilon_{d}} & \\
& -\frac{\varepsilon_{d} \Lambda_{p} h^{2}}{1+\varepsilon_{d}} & \frac{\Lambda_{p} h^{2}}{1+\varepsilon_{d}} & \frac{\varepsilon_{d} \Lambda_{p} h^{2}}{1+\varepsilon_{d}} & \\
& -\frac{1}{1+\varepsilon_{d}} & -\frac{1}{1+\varepsilon_{d}} & \frac{2+\varepsilon_{d}}{1+\varepsilon_{d}}+\Lambda_{p} h^{2} & -1 \\
\cdots & & & & \cdots
\end{array}\right] .
$$

\section{EXTENSION TO TWO DIMENSIONS}

Next, we illustrate how the above method can be applied to two-dimensional problems. Consider a square region of

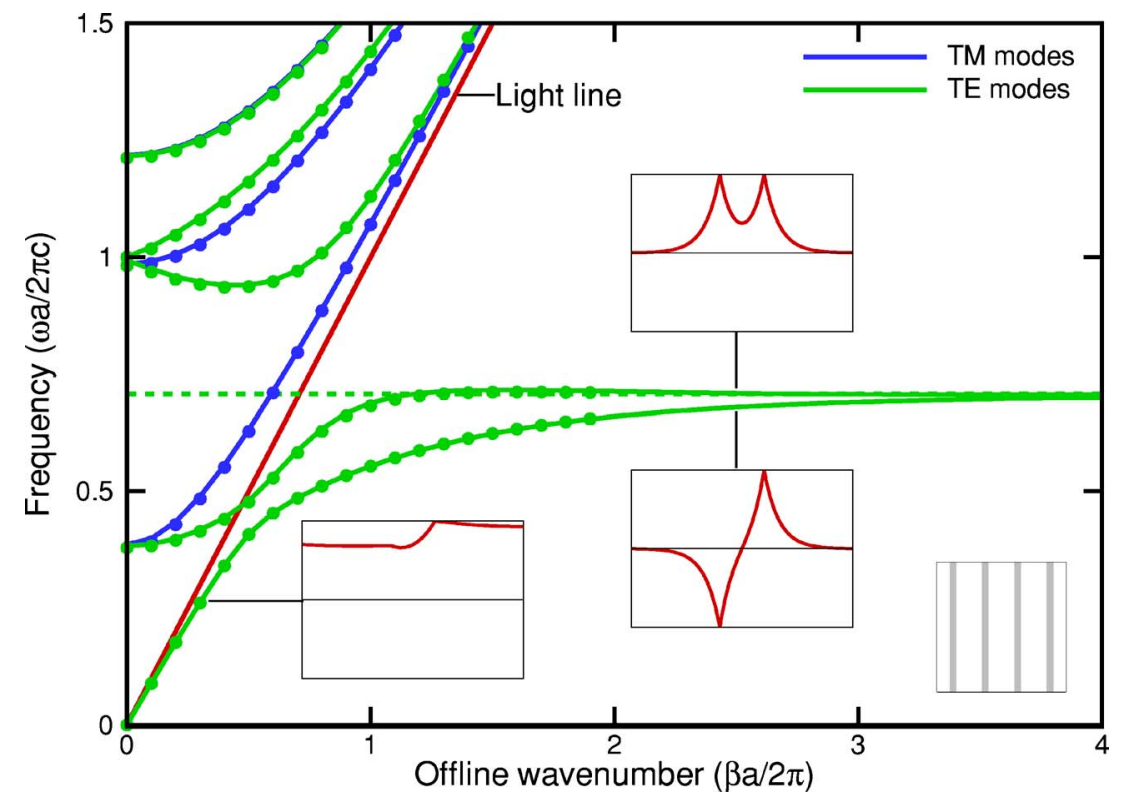

FIG. 4. (Color online) Dispersion relations at $k a / 2 \pi=0.1$ for the one-dimensional photonic crystal with $d_{2} / a=0.2$. 


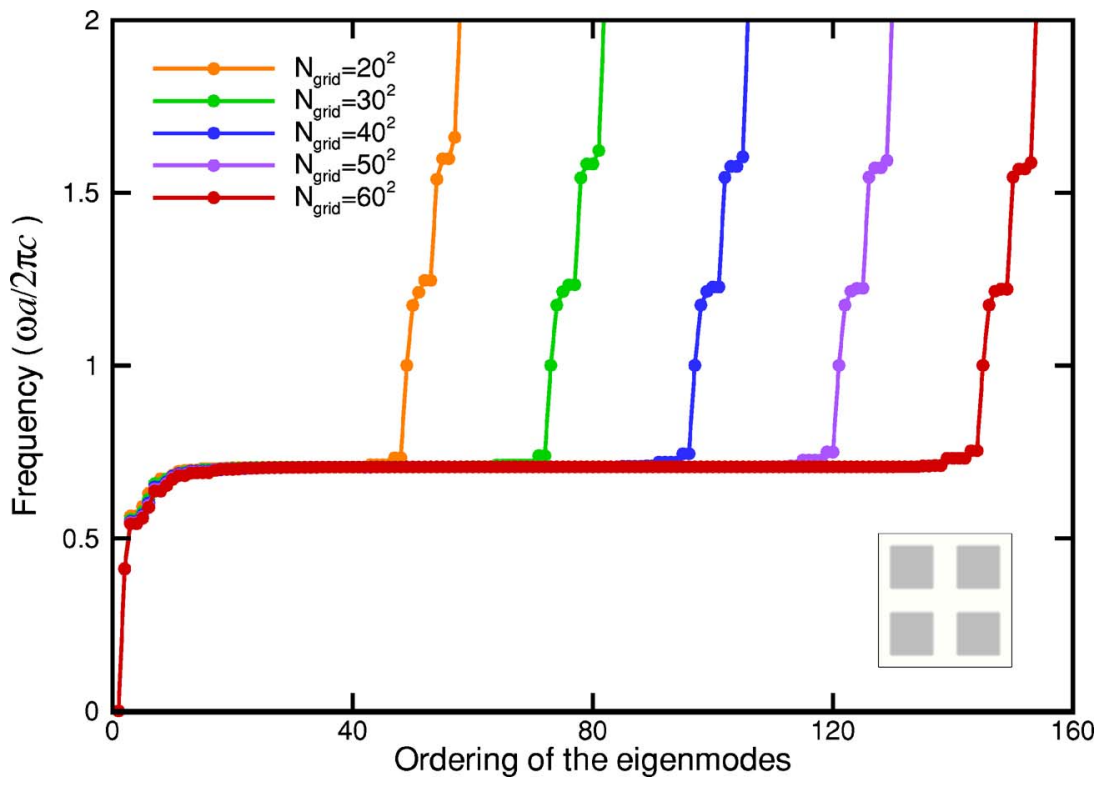

FIG. 5. (Color online) Eigenfrequencies at point $\Gamma$ for $\mathrm{TE}$ modes computed with different grid resolutions for the square lattice of square metallic cylinders with $w / a=0.3$.

metal surrounded by dielectrics as shown in Fig. 2. The interface condition (10) is applied in horizontal, vertical, and oblique directions. Therefore, in two dimensions there are two types of interface points: side and corner. At the interfacial point $S_{1}$ (side), now denoted as $(i, j)$, discretization of the interfacial operator is along $N_{1 L}-S_{1}-N_{1 R}$,

$$
\Lambda_{p}\left(\frac{H_{i, j}-H_{i-1, j}}{h}\right)=\Lambda\left[\frac{-H_{i-1, j}+\left(1+\varepsilon_{d}\right) H_{i, j}-\varepsilon_{d} H_{i+1, j}}{h}\right]
$$

and we define the interfacial variable as

$$
R_{i, j} \equiv-H_{i-1, j}+\left(1+\varepsilon_{d}\right) H_{i, j}-\varepsilon_{d} H_{i+1, j},
$$

or, reversely,

$$
H_{i, j}=\frac{H_{i-1, j}+R_{i, j}+\varepsilon_{d} H_{i+1, j}}{1+\varepsilon_{d}} .
$$

Then, the interface condition (10) for the interfacial point $S_{1}$ is

$$
\frac{\Lambda_{p}}{1+\varepsilon_{d}}\left(-\varepsilon_{d} H_{i-1, j}+R_{i, j}+\varepsilon_{d} H_{i+1, j}\right)=\Lambda R_{i, j}
$$

$R_{i, j}$ is defined similarly at the other interfacial points $S_{2}, S_{3}$, and $S_{4}$. At the interfacial point $C_{1}$ (corner), now denoted as $(i, j)$, discretization of the interfacial operator is along $A_{1}-C_{1}-N_{13}$

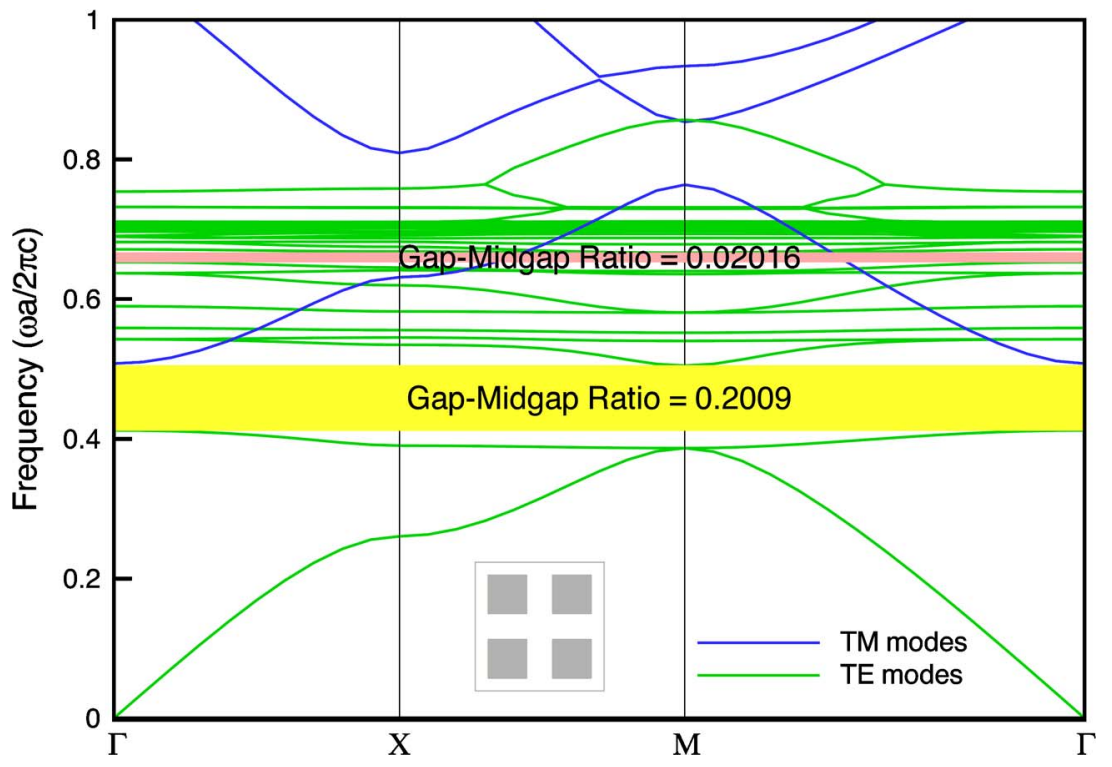

FIG. 6. (Color online) Band structures for the square lattice of square cylinders with half width $w / a=0.3$. 


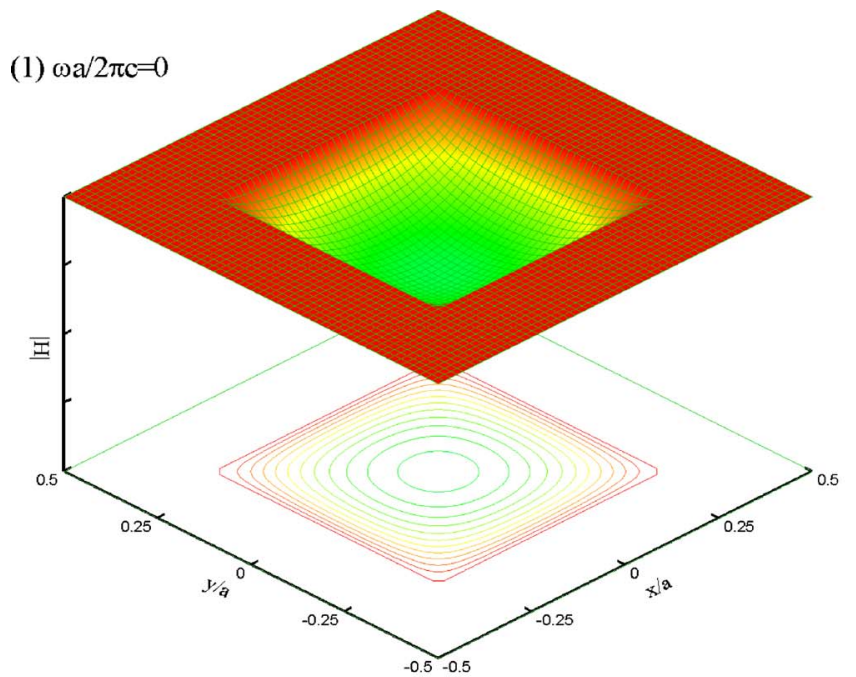

(3) $\omega \mathrm{a} / 2 \pi \mathrm{c}=0.5423$

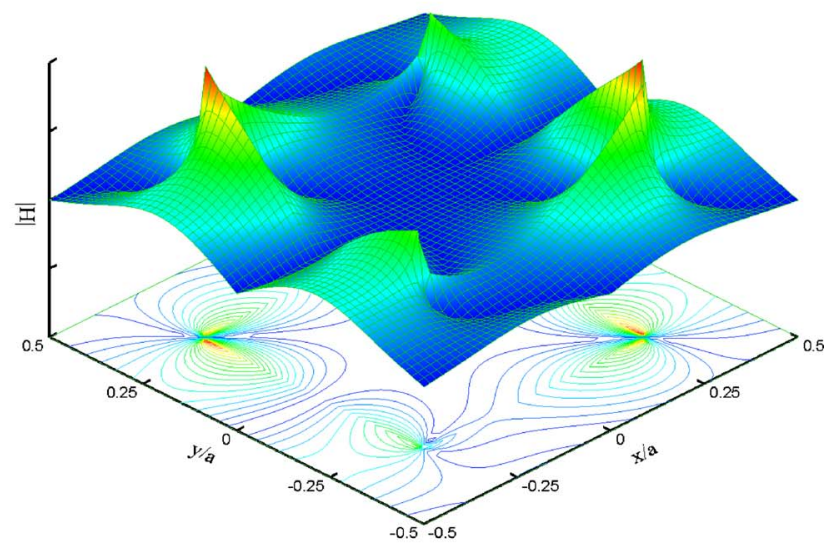

(2) $\omega \mathrm{a} / 2 \pi \mathrm{c}=0.4124$

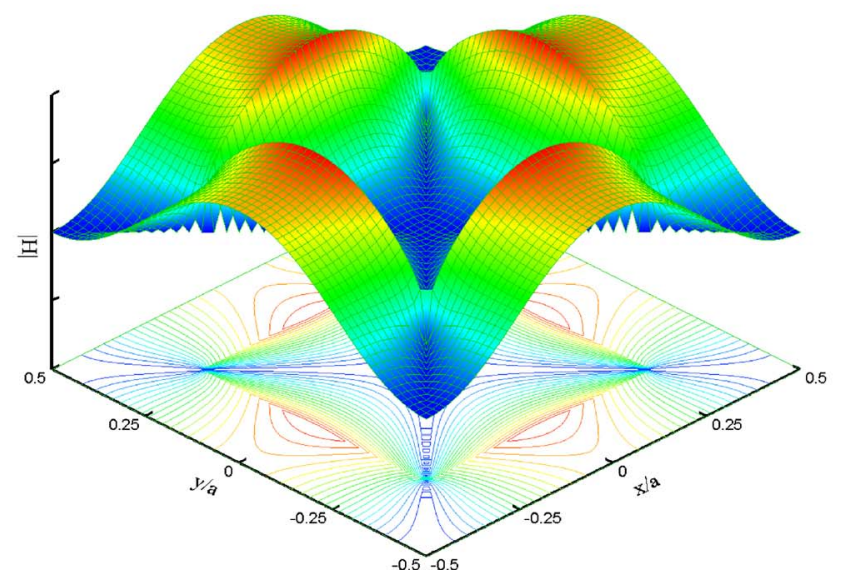

FIG. 7. (Color online) First to third TE modes in magnitude at point $\Gamma$ for the square lattice of square cylinders with half width $w / a=0.3$.

$$
\begin{aligned}
\Lambda_{p}( & \left.\frac{H_{i, j}-H_{i-1, j-1}}{\sqrt{2} h}\right) \\
& =\Lambda\left[\frac{-H_{i-1, j-1}+\left(1+\varepsilon_{d}\right) H_{i, j}-\varepsilon_{d} H_{i+1, j+1}}{\sqrt{2} h}\right],
\end{aligned}
$$

and the interfacial variable is defined as

$$
R_{i, j} \equiv-H_{i-1, j-1}+\left(1+\varepsilon_{d}\right) H_{i, j}-\varepsilon_{d} H_{i+1, j+1},
$$

or, reversely,

$$
H_{i, j}=\frac{H_{i-1, j-1}+R_{i, j}+\varepsilon_{d} H_{i+1, j+1}}{1+\varepsilon_{d}} .
$$

Then, the interface condition (10) for the interfacial point $C_{1}$ is

$$
\frac{\Lambda_{p}}{1+\varepsilon_{d}}\left(-\varepsilon_{d} H_{i-1, j-1}+R_{i, j}+\varepsilon_{d} H_{i+1, j+1}\right)=\Lambda R_{i, j} .
$$

$R_{i, j}$ is defined similarly at the other interfacial points $C_{2}, C_{3}$, and $C_{4}$. The finite-difference equations for the points next to the interface are obtained by replacing each $H_{i j}$ at the inter- face points with $R_{i j}$ through Eqs. (19) and (23), and the like equations. For example, for point $N_{1 L}$, central finite difference applied to Eq. (5) gives

$$
\frac{1}{\varepsilon_{d} h^{2}}\left(-H_{i-1, j}-H_{i, j-1}+4 H_{i, j}-H_{i+1, j}-H_{i, j+1}\right)=\Lambda H_{i} .
$$

Using Eq. (19) for $(i+1, j)$ to replace $H_{i+1, j}$ with $R_{i+1, j}$, we obtain

$$
\begin{gathered}
\frac{1}{\varepsilon_{d} h^{2}}\left[\left(4-\frac{1}{1+\varepsilon_{d}}\right) H_{i, j}-H_{i-1, j}-\frac{1}{1+\varepsilon_{d}} R_{i+1, j}\right. \\
\left.-\frac{\varepsilon_{d}}{1+\varepsilon_{d}} H_{i+2, j}-H_{i, j-1}-H_{i, j+1}\right]=\Lambda H_{i, j} .
\end{gathered}
$$

Also, for point $N_{5 L}$, using Eq. (23) for $(i+1, j)$ to replace $H_{i+1, j}$ with $R_{i+1, j}$, we obtain 
(4) $\omega \mathrm{a} / 2 \pi \mathrm{c}=0.5423$

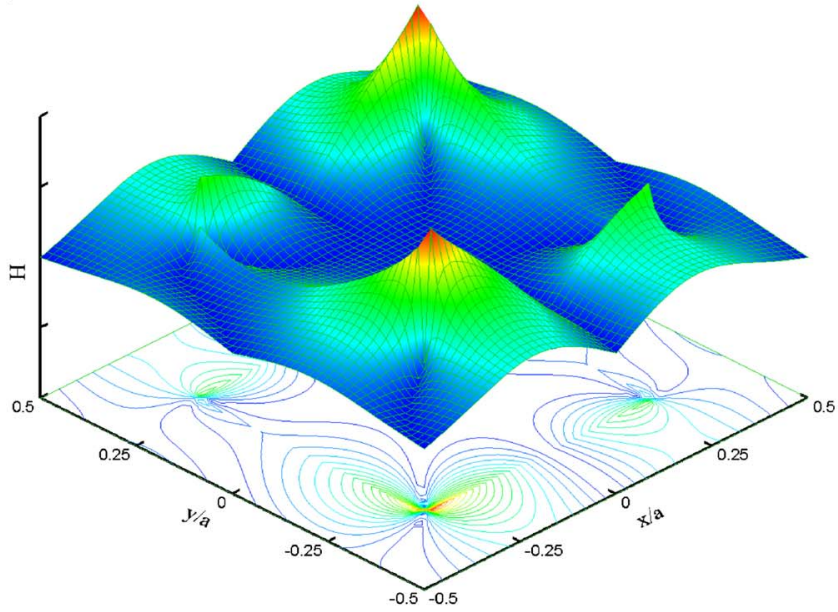

(5) $\omega \mathrm{a} / 2 \pi \mathrm{c}=0.5585$

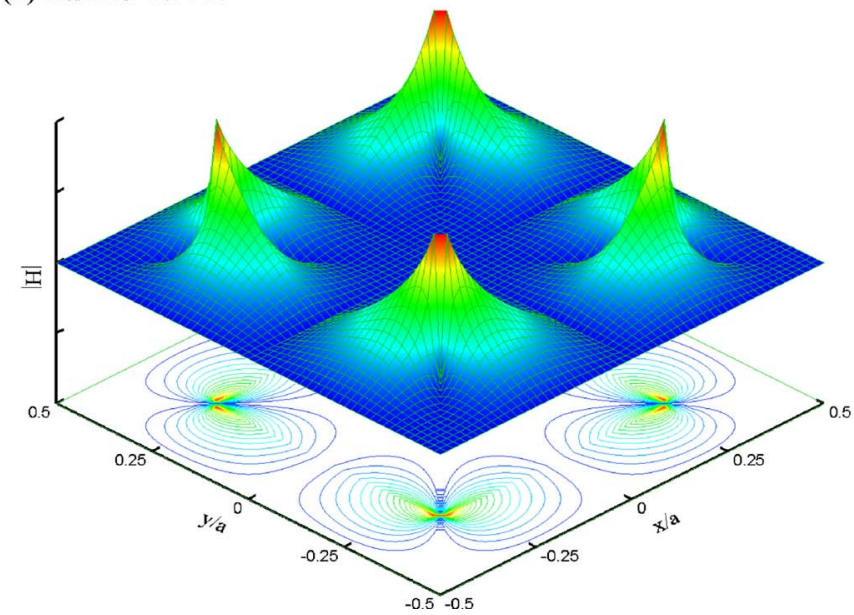

(6) $\omega \mathrm{a} / 2 \pi \mathrm{c}=0.5897$

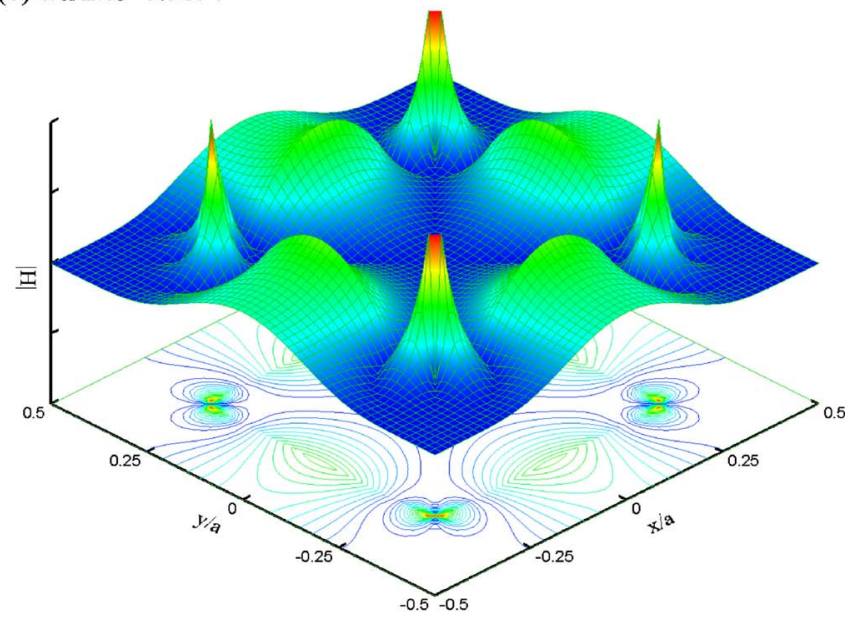

FIG. 8. (Color online) Fourth to sixth TE modes in magnitude at point $\Gamma$ for the square lattice of square cylinders with half width $w / a=0.3$.

$$
\begin{gathered}
\frac{1}{\varepsilon_{d} h^{2}}\left[4 H_{i, j}-H_{i-1, j}-\frac{1}{1+\varepsilon_{d}} R_{i+1, j}-\frac{\varepsilon_{d}}{1+\varepsilon_{d}} H_{i+2, j+1}\right. \\
\left.-\left(1+\frac{1}{1+\varepsilon_{d}}\right) H_{i, j-1}-H_{i, j+1}\right]=\Lambda H_{i, j},
\end{gathered}
$$

and, for point $N_{13}$, central finite difference applied to Eq. (6) gives

$$
\frac{1}{h^{2}}\left[-H_{i-1, j}-H_{i, j-1}+\left(4+\Lambda_{p} h^{2}\right) H_{i, j}-H_{i+1, j}-H_{i, j+1}\right]=\Lambda H_{i} .
$$

Using similar equations for $(i-1, j)$ and $(i, j-1)$ to replace $H_{i-1, j}$ and $H_{i, j-1}$ with $R_{i-1, j}$ and $R_{i, j-1}$, we obtain

$$
\begin{aligned}
\frac{1}{h^{2}} & \left(4-\frac{2 \varepsilon_{d}}{1+\varepsilon_{d}}+\Lambda_{p}\right) H_{i, j}-\frac{1}{1+\varepsilon_{d}} H_{i-2, j}-\frac{1}{1+\varepsilon_{d}} R_{i-1, j} \\
& \left.-H_{i+1, j}-\frac{1}{1+\varepsilon_{d}} H_{i, j-2}-\frac{1}{1+\varepsilon_{d}} R_{i, j-1}-H_{i, j+1}\right]=\Lambda H_{i, j} .
\end{aligned}
$$

The other neighboring points can be obtained in a similar manner. Along with the modifications for the neighboring points described above, the resultant matrix is then obtained by replacing $H_{i, j}$ at all interface points with $R_{i, j}$ through Eqs. (20) and (24), and the like equations.

\section{RESULTS AND DISCUSSION}

In order to justify the present approach, we report the results in one-dimensional photonic crystals, which analytical solutions of band structures are available. Consider a photonic crystal with lattice constant $a$. The widths of the dielectric and the metal are $d_{1}$ and $d_{2}$, respectively. Then, according to Eqs. (5) and (6), we have the equations for the $H$ fields in the dielectric (denoted as $H_{+}$) and the metal (denoted as $\left.H_{-}\right)$, respectively, as

$$
\left\{\begin{array}{l}
H_{+}^{\prime \prime}+k_{1}^{2} H_{+}=0,0 \leqslant x \leqslant d_{1}, \\
H_{-}^{\prime \prime}+k_{2}^{2} H_{-}=0, d_{1} \leqslant x \leqslant a,
\end{array}\right.
$$

where $k_{1}^{2}=(\omega / c)^{2} \varepsilon_{d}-\beta^{2}$ and $k_{2}^{2}=(\omega / c)^{2} \varepsilon_{m}-\beta^{2}$ are the inplane wave numbers in the dielectric and the metal, respec- 
(7) $\omega \mathrm{a} / 2 \pi \mathrm{c}=0.637$

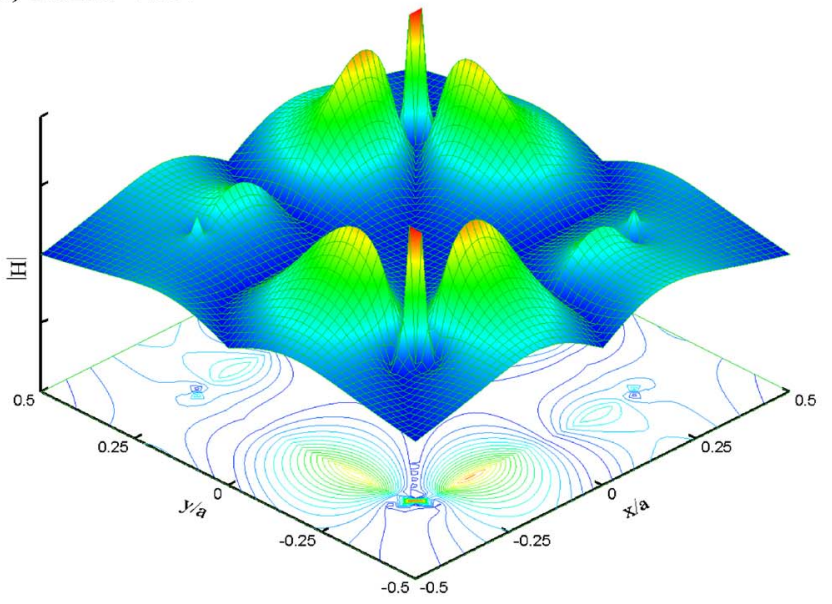

(8) $\omega \mathrm{a} / 2 \pi \mathrm{c}=0.637$

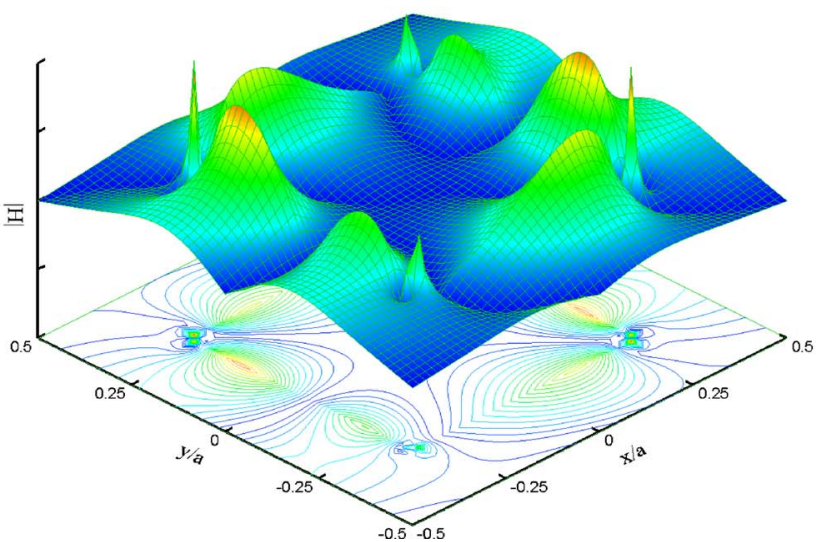

(9) $\omega \mathrm{a} / 2 \pi \mathrm{c}=0.6531$

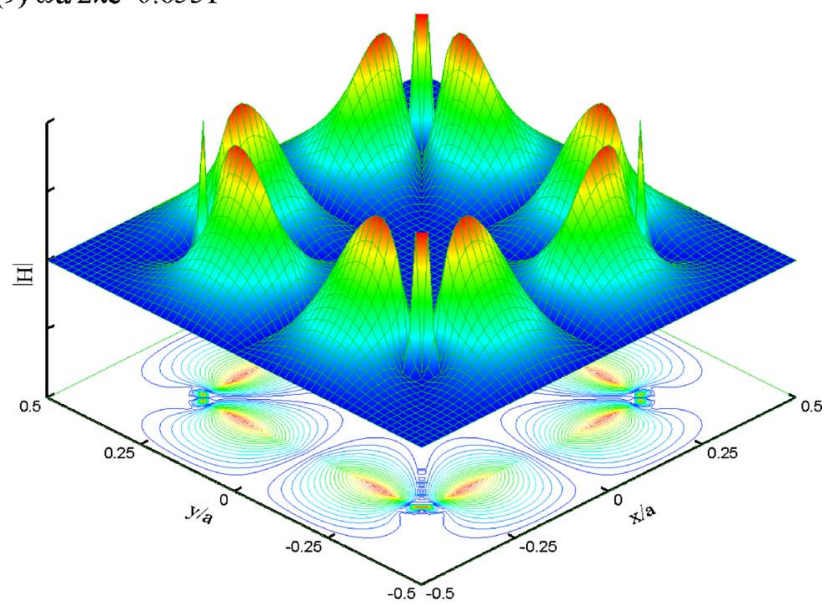

FIG. 9. (Color online) Seventh to ninth TE modes in magnitude at point $\Gamma$ for the square lattice of square cylinders with half width $w / a=0.3$.

tively, and $\beta$ is the off-plane wave number. Assume the solutions have the form

$$
\left\{\begin{array}{l}
H_{+}(x)=A e^{i k_{1} x}+B e^{-i k_{1} x}, 0 \leqslant x \leqslant d_{1}, \\
H_{-}(x)=C e^{i k_{2} x}+D e^{-i k_{2} x}, d_{1} \leqslant x \leqslant a,
\end{array}\right.
$$

where $A, B, C$, and $D$ are the weighting coefficients to be determined by using the continuity conditions at the interface

$$
\left\{\begin{array}{l}
H_{+}\left(d_{1}\right)=H_{-}\left(d_{1}\right), \\
\varepsilon_{m} H_{+}^{\prime}\left(d_{1}\right)=\varepsilon_{d} H_{-}^{\prime}\left(d_{1}\right),
\end{array}\right.
$$

and the Bloch conditions at the domain boundary

$$
\left\{\begin{array}{l}
H_{+}(0)=e^{-i k d} H_{-}(a) \\
\varepsilon_{m} H_{+}^{\prime}(0)=\varepsilon_{d} e^{-i k d} H_{-}^{\prime}(a) .
\end{array}\right.
$$

In order to obtain a nontrivial solution, the determinant of the $4 \times 4$ matrix has to be zero

$$
\left|\begin{array}{cccc}
1 & 1 & -e^{i\left(k_{2}-k\right) a} & -e^{-i\left(k_{2}+k\right) a} \\
\frac{k_{1}}{\varepsilon_{d}} & -\frac{k_{1}}{\varepsilon_{d}} & -\frac{k_{2}}{\varepsilon_{m}} e^{i\left(k_{2}-k\right) a} & \frac{k_{2}}{\varepsilon_{m}} e^{-i\left(k_{2}+k\right) a} \\
e^{i k_{1} d_{1}} & e^{-i k_{1} d_{1}} & -e^{i k_{2} d_{1}} & -e^{-i k_{2} d_{1}} \\
\frac{k_{1}}{\varepsilon_{d}} e^{i k_{1} d_{1}} & -\frac{k_{1}}{\varepsilon_{d}} e^{-i k_{1} d_{1}} & -\frac{k_{2}}{\varepsilon_{m}} e^{i k_{2} d_{1}} & \frac{k_{2}}{\varepsilon_{m}} e^{-i k_{2} d_{1}}
\end{array}\right|=0,
$$

which can be rearranged to obtain the analytical formula ${ }^{15}$

$$
\begin{aligned}
\cos (k a) & =\cos \left(k_{1} d_{1}\right) \cos \left(k_{2} d_{2}\right) \\
- & \frac{1}{2}\left(\frac{\varepsilon_{m} k_{1}}{\varepsilon_{d} k_{2}}+\frac{\varepsilon_{d} k_{2}}{\varepsilon_{m} k_{1}}\right) \sin \left(k_{1} d_{1}\right) \sin \left(k_{2} d_{2}\right) .
\end{aligned}
$$

Next, we present the results in two dimensions. In the following discussion, we take $\varepsilon_{d}=1$ (air). Let $\omega_{\text {sp }}$ denote the surface plasma frequency for a single flat interface between the metal and the air $\left(\omega_{\mathrm{sp}}=\omega_{p} / \sqrt{2}\right)$, and here the normalized $\omega_{p} a / 2 \pi c$ is taken to be unity. In particular, we are interested in the dependence of the modes of surface plasmon polariton on the off-line wave number $\beta$ at the zone center $k=0$. Figure 3 shows the computed results by the interfacial operator 
(10) $\omega \mathrm{a} / 2 \pi \mathrm{c}=0.6712$

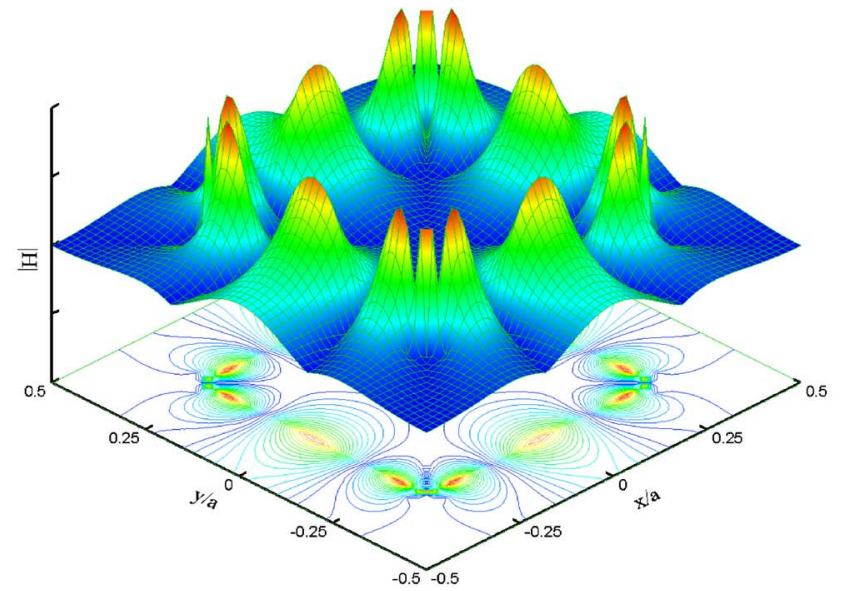

(11) $\omega \mathrm{a} / 2 \pi \mathrm{c}=0.6817$

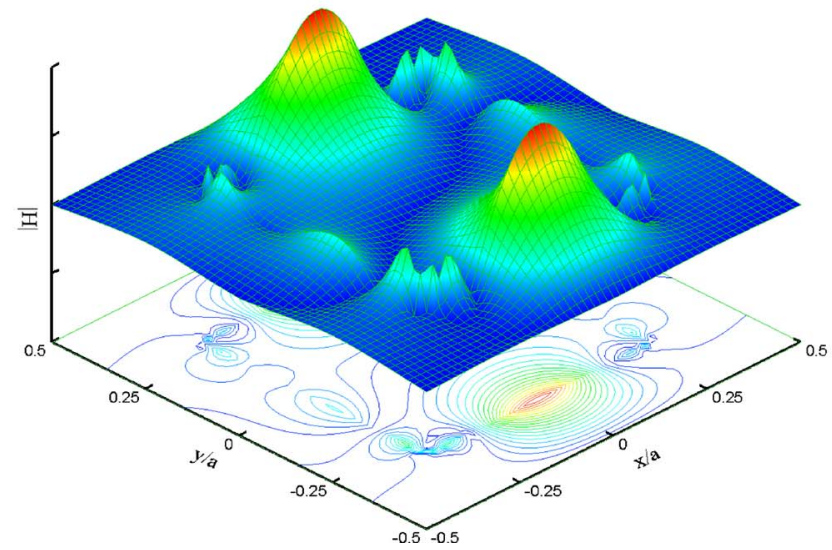

(12) $\omega \mathrm{a} / 2 \pi \mathrm{c}=0.6817$

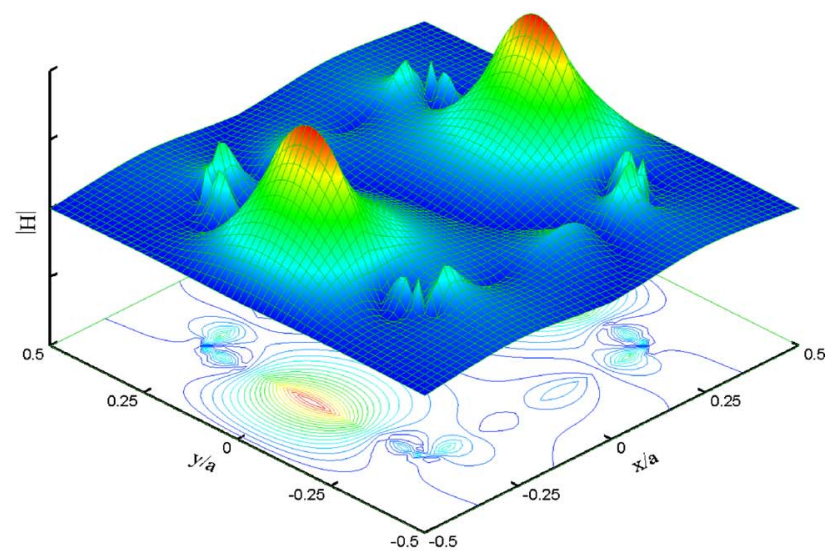

FIG. 10. (Color online) Tenth to twelfth TE modes in magnitude at point $\Gamma$ for the square lattice of square cylinders with half width $w / a=0.3$.

formulation, which are in very close agreement with the ones obtained by applying the Newton-Ralphson method ${ }^{6}$ to the analytical formula (35). In addition to the lower branch of surface plasmon polariton, there is an upper branch that also converges to the surface plasma frequency $\omega_{\text {sp }}$ in the large $\beta$ limit. In the case of thin metallic components, interaction of surface plasmon polaritons on both sides leads to mode splitting: one lower frequency with odd symmetry and one higher frequency with even symmetry. In the case of thick metallic components, the upper branch corresponds to collective oscillations of electrons in the metal; this can be observed by increasing the thickness of the metal layer (small air gap) that the upper branch at $k=0$ shifts to a higher frequency, and eventually to the plasma frequency $\omega_{p}$. Figure 4 is a similar plot at $k \neq 0$. The difference between Figs. 3 and 4 is that at small off-line wave-number $\beta$, the first mode is symmetric for $k=0$ and is antisymmetric for $k \neq 0$. Both of the first two TE bands tend to have the surface plasma frequency $\omega_{s p} a / 2 \pi c=1 / \sqrt{2}$. However, it is known that at large off-line wave-number $\beta$, the lower frequency mode has odd symmetry, while the higher frequency has even symmetry. ${ }^{16}$ Therefore, there is a crossing for $k=0$ (Fig. 3), while we would expect the anticrosssing scheme for $k \neq 0$. Moreover, the periodic array of metals can be considered as metal gratings that cause crossing of the light line by the second TE band. ${ }^{17}$

Next, we turn to the square lattice with square metallic components as in Fig. 2 with half width $w / a=0.3$. It has been suggested that the number of modes of surface plasmon polariton is infinite. ${ }^{8}$ To test this hypothesis, we solve the eigenvalue problem (15) with a different number of grid points, which can also help check the dependence of modes on the grid resolution. Figure 5 presents the results that (i) for the mode with a frequency lower or greater than the surface plasma frequency $\omega_{\mathrm{sp}}$, the mode is little dependent on the grid resolution as long as the number of grid points is not less then $30 \times 30$ and (ii) there is an increasing number of stationary (dispersionless) modes near the resonant frequency $\omega_{\text {sp }}$ with increasing the grid resolution. Figure 5 seems to indicate that the number of stationary modes increases with a rate proportional to the linear resolution of the grid, i.e., with $N$, if the number of grid points is $N \times N$.

Figure 6 shows the band structures computed by the interfacial operator approach. Unlike TE modes, the lowest branch of TM modes has a cut-off frequency even at small filling ratios of the metal, and the cut-off frequency shifts further upward with increasing the filling ratio, leaving a large full photonic band gap that is solely determined by the TE modes. From the fourth branch of modes, the dispersion 
$\omega \mathrm{a} / 2 \pi \mathrm{c}=0.707$

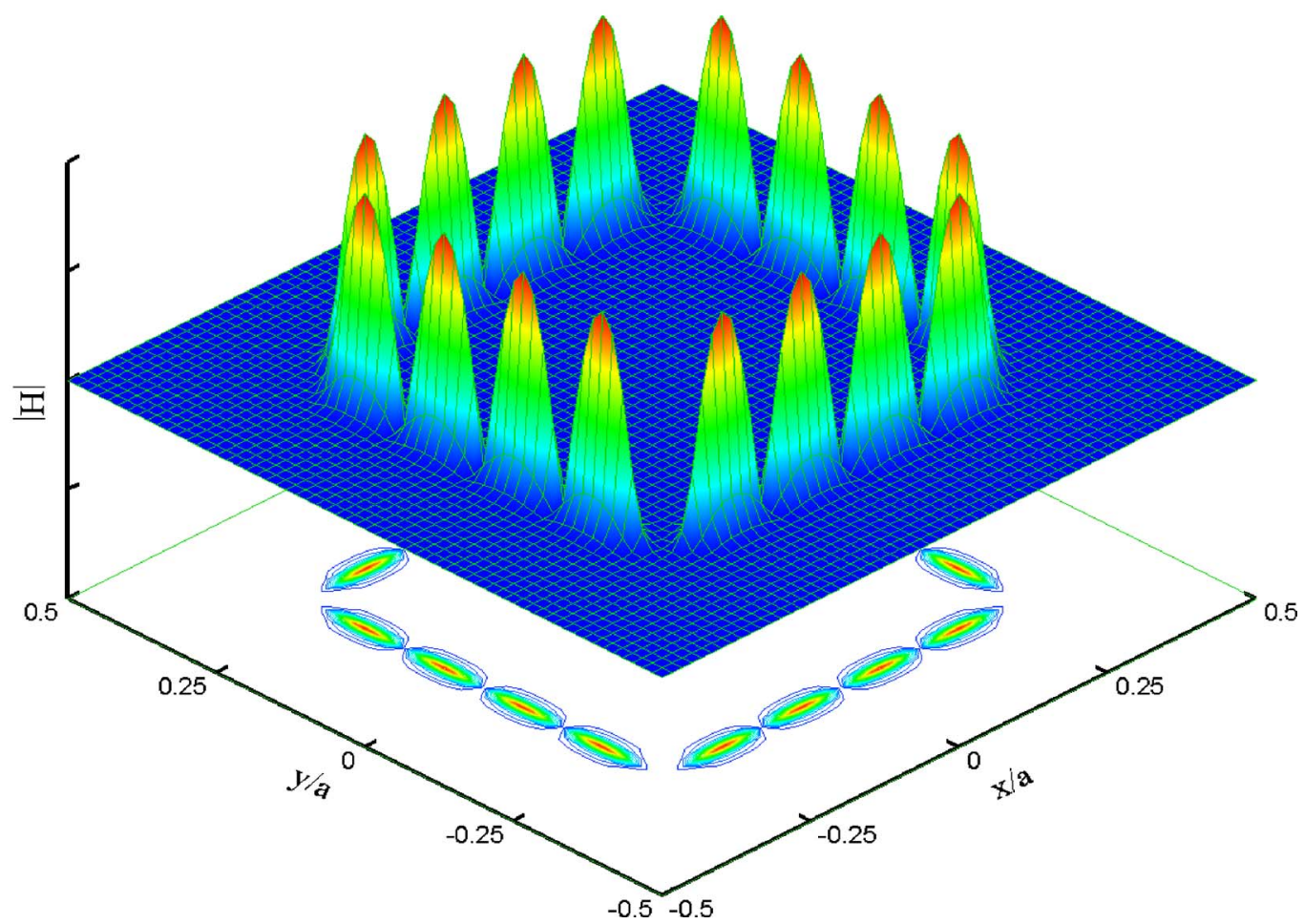

FIG. 11. (Color online) One of the highly localized TE modes near the frequency $\omega_{\text {sp }}$ for the square lattice of square cylinders with half width $w / a=0.3$.

relation for the TE modes is each a quite flat one, denoting standing modes for frequencies up to the surface plasma frequency $\omega_{\mathrm{sp}}$. A small band gap is observed to exist between these flat bands, and is qualified for the name of plasmonic band gap. The gap is larger at even larger filling ratios. Figures 7-10 show the first $12 \mathrm{TE}$ modes for the square lattice in Fig. 2 at the point $\Gamma$. All these modes have a frequency lower than the surface plasma frequency $\omega_{\mathrm{sp}}$, but as we can see, each mode concentrates more or less on the neighborhood of the interface between the metal and the dielectric. It is noted that some of these modes are degenerate, e.g., the third and the fourth modes, the seventh and eightth modes, and the eleventh and twelfth modes. Figure 11 shows an example of highly localized nature of surface plasmon polariton (at $\left.\omega_{\mathrm{sp}}\right)$, living in a close neighborhood of the interface. As the frequency exceeds the plasma frequency $\omega_{p}$, the typical TE mode is highly oscillatory (Fig. 12).

As a final example, we examine the effect of (regular) roughness on the modes of surface plasmon polariton. Figure 13 shows the band structures for the square lattice with thinner wavy metallic components with amplitude $r / a=0.1$ and thickness $t / a=0.1$. The most significant feature is widely spread flat bands of stationary modes with frequencies well exceeding the surface plasma frequency. This flat-band broadening was not observed for the square lattice with square or circular metallic components. The physical origin of this broadening could be explained as effective mode interaction due to the geometry of the interface. The modes of surface plasmon polariton interact with each other from both the normal and the lateral directions, further lifting the de- generacy. The broadening of the flat bands may be particularly useful for those applications which exploit modes of surface plasmon polarition such as surface enhanced Raman scattering, ${ }^{18}$ and conceivably solar cells. ${ }^{4}$ As we increase the thickness of the metallic components, the broadening becomes less significant with most flat-band frequencies below the surface plasma frequency $\omega_{s p}$ (Fig. 14). Nevertheless, densely distributed bands exist with frequencies substantially above the surface plasma frequency $\omega_{\mathrm{sp}}$, leaving a significant plasmonic band gap.

The present method can be extended in order to take into account the Drude damping in the dielectric function, which comes from the collisions of the electrons. For this purpose, we introduce the Drude model

$$
\varepsilon(\omega)=1-\frac{\omega_{p}^{2}}{\omega^{2}+i \gamma \omega},
$$

where $\gamma$ is the collision frequency of free electrons. If the damping is small such that $\gamma \ll \omega$, then the damped solutions can be regarded as perturbations to the undamped solutions for $\gamma=0$ (the free-electron model). First, the operator $L$ of the eigensystem $L u=\Lambda u$ is split into two terms

$$
L=L_{0}+L_{1}
$$

where $L_{0}$ is the operator of the undamped eigensystem. Let $\Lambda^{(0)}$ denote the eigenvalue of $L_{0}$, and assume the solution for the operator $L$ is expanded as 


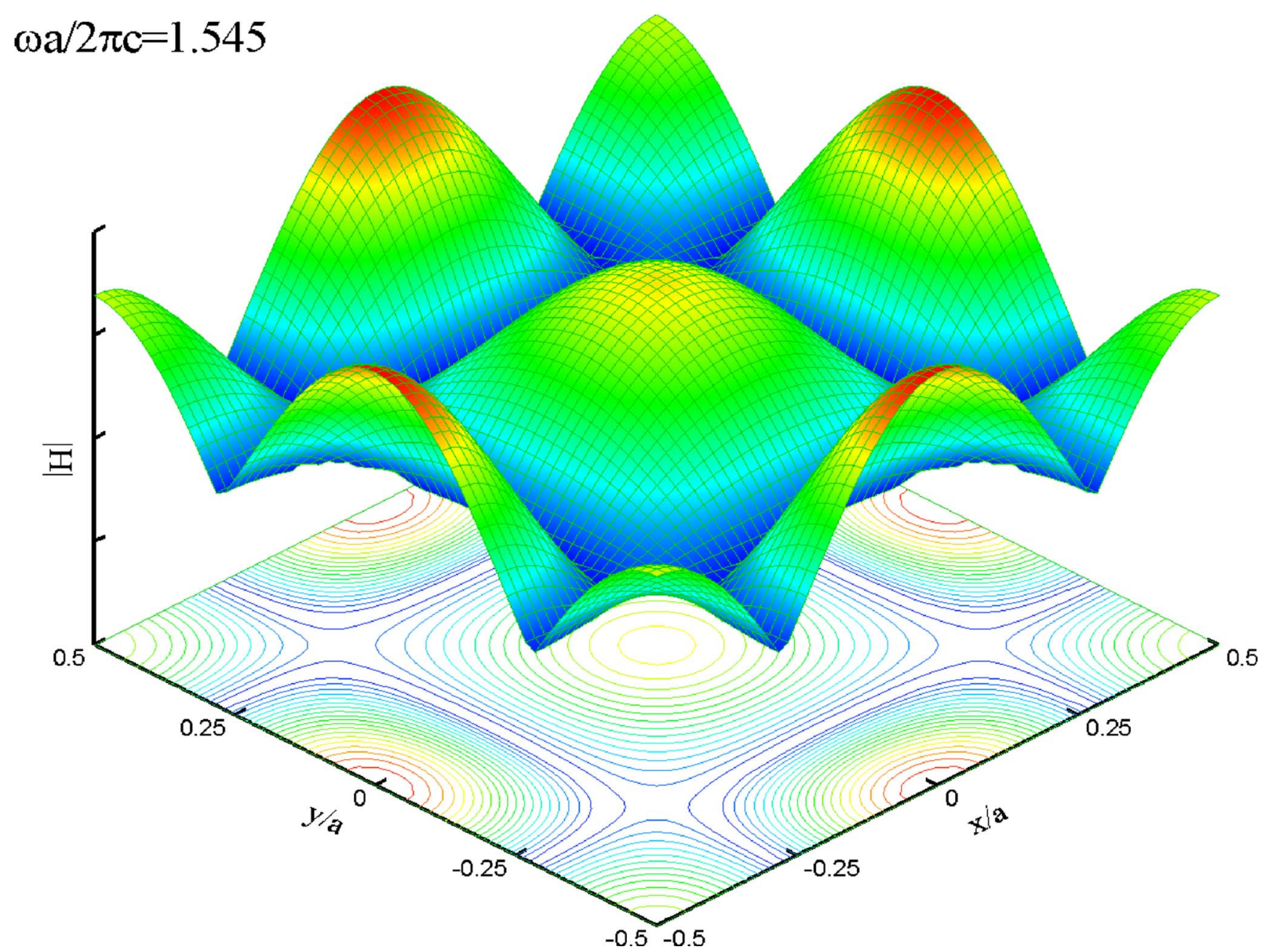

FIG. 12. (Color online) One of the highly oscillatory TE modes above the frequency $\omega_{\mathrm{sp}}$ for the square lattice of square cylinders with half width $w / a=0.3$.

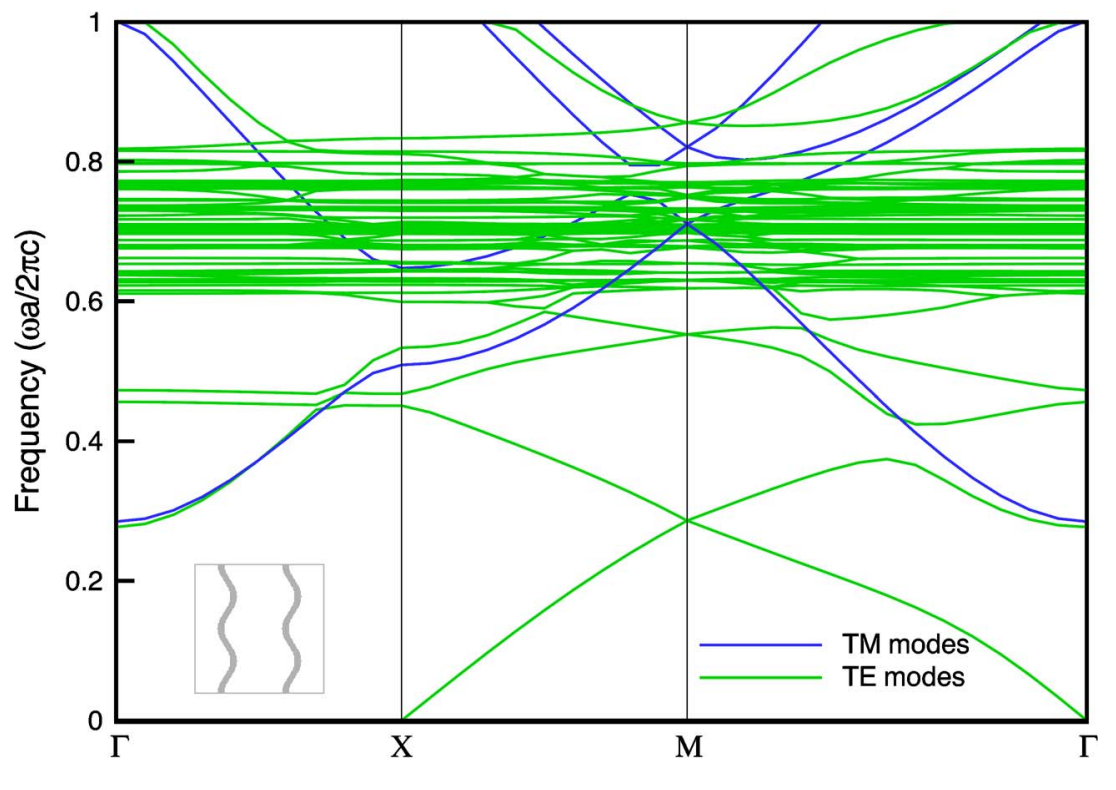

FIG. 13. (Color online) Band structures for the square lattice of wavy structure with thickness $t / a$ $=0.1$ and amplitude $r / a=0.1$. 


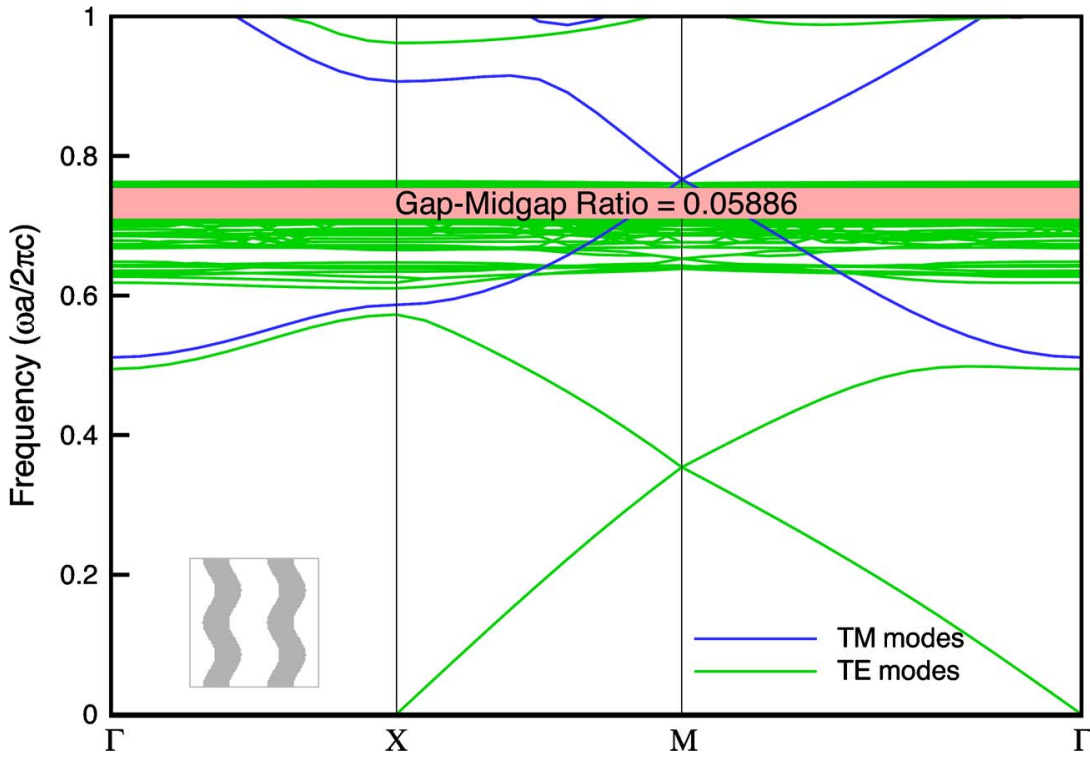

FIG. 14. (Color online) Band structures for the square lattice of wavy structure with thickness $t / a=0.4$ and amplitude $r / a=0.1$.

$$
\Lambda=\Lambda^{(0)}+\Lambda^{(1)}+\cdots
$$

Based on the interfacial operator within the finite-difference framework, we have

$$
\begin{gathered}
L H_{i}=\frac{1}{\varepsilon_{d} h^{2}}\left(-H_{i-1}+2 H_{i}-H_{i+1}\right), \\
L_{0} H_{i}=\frac{1}{\varepsilon_{d} h^{2}}\left(-H_{i-1}+2 H_{i}-H_{i+1}\right), \\
L_{1}=0,
\end{gathered}
$$

for points in the dielectric

$$
L H_{i}=\frac{1}{h^{2}}\left[-H_{i-1}+\left(2+\frac{\Lambda_{p} h^{2}}{1+i \gamma / \omega}\right) H_{i}-H_{i+1}\right],
$$

$$
L_{0} H_{i}=\frac{1}{h^{2}}\left[-H_{i-1}+\left(2+\Lambda_{p} h^{2}\right) H_{i}-H_{i+1}\right],
$$

$$
L_{1} H_{i}=-\Lambda_{p}\left(\frac{i \gamma}{\omega+i \gamma}\right) H_{i},
$$

for points in the metal, and

$$
\begin{gathered}
L R_{i}=\left(-\frac{\varepsilon_{d} \Lambda_{p}}{1+\varepsilon_{d}} H_{i-1}+\left(\frac{\Lambda_{p}}{1+\varepsilon_{d}}-i \gamma \omega\right) R_{i}+\frac{\varepsilon_{d} \Lambda_{p}}{1+\varepsilon_{d}} H_{i+1}\right), \\
L_{0} R_{i}=\left(-\frac{\varepsilon_{d} \Lambda_{p}}{1+\varepsilon_{d}} H_{i-1}+\frac{\Lambda_{p}}{1+\varepsilon_{d}} R_{i}+\frac{\varepsilon_{d} \Lambda_{p}}{1+\varepsilon_{d}} H_{i+1}\right),
\end{gathered}
$$

$$
L_{1} R_{i}=-i \gamma \omega R_{i},
$$

for points at the interface. With this operator splitting, we can refer to standard perturbation theory ${ }^{11-14}$ to obtain the first-

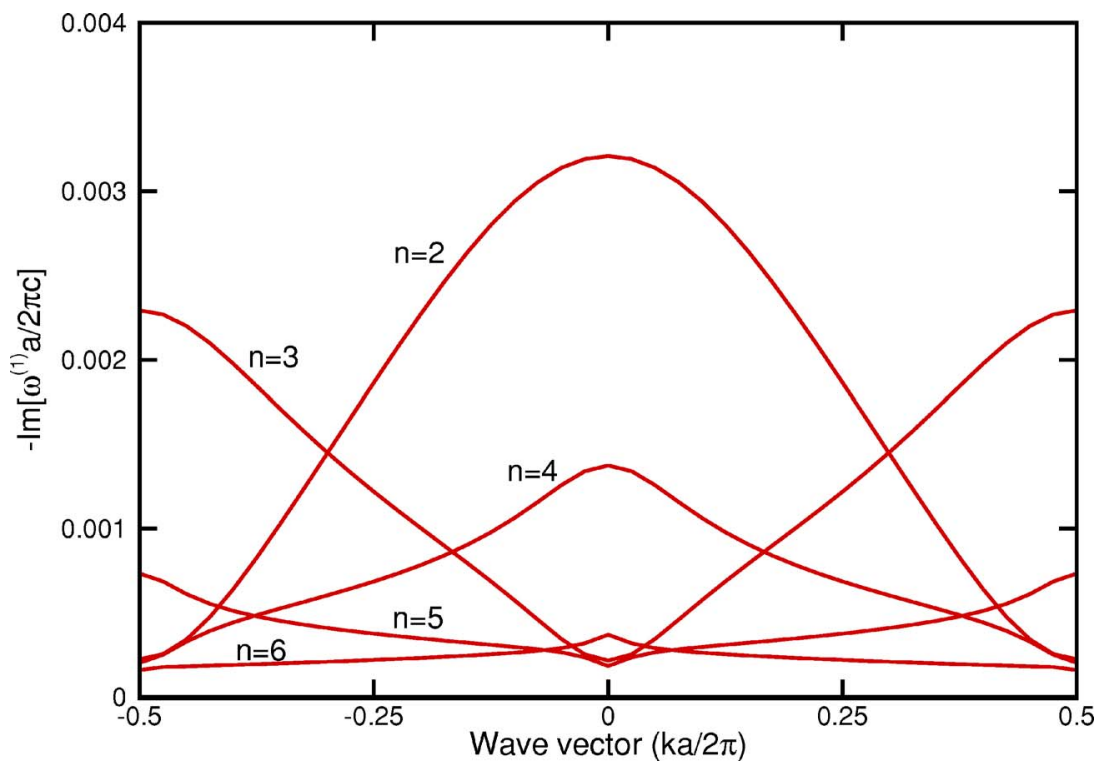

FIG. 15. (Color online) Imaginary part of $\omega^{(1)}$ for first few bands of the one-dimensional photonic crystal with $d_{2} / a=0.2$ and $\gamma / \omega_{p}=0.01$. 
order correction of the eigenvalue $\Lambda^{(1)}$. The correction of the eigenfrequency $\omega^{(1)}$ is given as

$$
\omega^{(1)}=c\left(\sqrt{\Lambda^{(0)}+\Lambda^{(1)}}-\sqrt{\Lambda^{(0)}}\right) .
$$

Figure 15 shows the imaginary part of $\omega^{(1)}$ of the first few bands for a one-dimensional photonic crystal with $d_{2} / a$ $=0.2$ and $\gamma / \omega_{p}=0.01$. It is noted that even bands have larger dissipations at the zone center, while odd bands have larger dissipations at the band edges. Besides, the dissipation becomes smaller for higher bands.

\section{CONCLUDING REMARKS}

In conclusion, the present approach presents a direct eigenvalue problem for computing the modes of surface plasmon polariton as well as the higher frequency modes. In particular, we have considered the free-electron model and the model of Drude damping for the metal. Several interest- ing phenomena have been uncovered and discussed; these include the anticrossing scheme for one-dimensional array, the number of TE modes at the resonant frequency, the qualification of plasmonic band gap, and band broadening for two-dimensional periodic structures. Nevertheless, in the wide range of energy spectrum: $0 \sim 2 \hbar \omega_{p}$, the contribution of interband transitions is not negligible. ${ }^{19,20}$ This proposes a further challenge for the present methodology because the contribution of the interband transitions lacks a simple functional form in frequency. The issue is currently under investigation.

\section{ACKNOWLEDGMENTS}

This work was supported in part by National Science Council of the Republic of China under Contract No. NSC 91-2212-E-002-072, and the Ministry of Economic Affairs of the Republic of China under Contract No. MOEA 93-EC-17A-08-S1-0006.
*Electronic address: mechang@gate.sinica.edu.tw

${ }^{1}$ R. H. Ritchie, Phys. Rev. 106, 874 (1957).

${ }^{2}$ J. B. Pendry, L. Martin-Moreno, and F. J. Garcia-Vidal, Science 305, 847 (2004).

${ }^{3}$ S. C. Kitson, W. L. Barnes, and J. R. Sambles, Phys. Rev. Lett. 77, 2670 (1996).

${ }^{4}$ W. L. Barnes, A. Dereux, and T. W. Ebbesen, Nature (London) 424, 824 (2003).

${ }^{5}$ O. Toader and S. John, Phys. Rev. E 70, 046605 (2004).

${ }^{6}$ D. Kincaid and W. Cheney, Numerical Analysis, 2nd ed. (Brooks/ Cole, Pacific Grove, 1996).

${ }^{7}$ E. Moreno, D. Erni, and C. Hafner, Phys. Rev. B 65, 155120 (2002).

${ }^{8}$ T. Ito and K. Sakoda, Phys. Rev. B 64, 045117 (2001).

${ }^{9}$ A. Tip, A. Moroz, and J. M. Combes, J. Phys. A 33, 6223 (2000).

${ }^{10}$ A. Moroz, A. Tip, and J. M. Combes, Synth. Met. 116, 481 (2001).

${ }^{11}$ H. van der Lem, A. Tip, and A. Moroz, J. Opt. Soc. Am. B 20,
1334 (2003).

${ }^{12}$ J. M. Combes, B. Gralak, and A. Tip, Contemp. Math. 339, 1 (2003).

${ }^{13}$ K. C. Huang, P. Bienstman, J. D. Joannopoulos, K. A. Nelson, and S. Fan, Phys. Rev. Lett. 90, 196402 (2003).

${ }^{14}$ C. C. Chang, J. Y. Chi, R. L. Chern, C. C. Chang, C. H. Lin, and C. O. Chang, Phys. Rev. B 70, 075108 (2004).

${ }^{15} \mathrm{P}$. Yeh, Optical Waves in Layered Media (Wiley, New York, 1988).

${ }^{16}$ B. E. Sernelius, Surface Modes in Physics (Wiley, New York, 2001), p. 125.

${ }^{17}$ W.-C. Tan, T. W. Preist, J. R. Sambles, and N. P. Wanstall, Phys. Rev. B 59, 12661 (1999).

${ }^{18}$ M. Fleischmann, P. J. Hendra, and A. J. McQuillan, Chem. Phys. Lett. 26, 163 (1974).

${ }^{19}$ P. B. Johnson and R. W. Christy, Phys. Rev. B 6, 4370 (1972).

${ }^{20}$ A. Pinchuk, U. Kreibig, and A. Hilger, Surf. Sci. 557, 269 (2004). 